\title{
Resolution
}

\section{An Appeal to Save Sonneberg Observatory}

The participants of the IAU Colloquium No. 151 on "Flares and Flashes", held in Sonneberg December 5-9, 1994, express their utmost concern about the threat of closure of the Sonneberg Observatory as a research institute by the end of this year. Sonneberg is the only place in the world where routine sky patrol observations are being performed and such a step should be a dramatic loss for astronomical research which can never be recovered in the future. The participants are aware that a continuation of a sky patrol in the traditional form, on the basis of photographic plates, is neither feasible (for reasons of the imminent discontinuation of the production of such plates), nor practical, as technologically much more advanced detectors (CCDs) are readily available. They consider the ASPA project - "All Sky Patrol Astrophysics" - as the best means of efficient continuation, and in fact extension, of sky patrols with new technologies. In particular they consider the Sonneberg Observatory a most adequate site for the logistical center needed for ASPA, in view of the long tradition and expertise in this field, the large unique plate archive, and the deep involvement in high-technology research at the Technical University of Ilmenau. In view of this, the participants of IAU Colloquium No. 151 urge all involved local authorities to provide for the continuation of the scientific work at Sonneberg Observatory.

The participants of IAU Colloquium 151 "Flares and Flashes" 